\title{
Comparison of Vaginal Natural Orifice Transluminal Endoscopic Surgery (vNOTES) and Laparoendoscopic Single-Site (LESS) Hysterectomy on Postoperative Pain Reduction: A Randomized Pilot Study
}

\author{
Soo Jin Park · Hee Seung Kim · Ga Won Yim
}

Received: June 19, 2021 / Accepted: July 23, 2021 / Published online: August 10, 2021

(C) The Author(s) 2021

\begin{abstract}
Introduction: Vaginal natural orifice transluminal endoscopic surgery (vNOTES) hysterectomy has shown benefit in postoperative pain and operation time compared to laparoscopic hysterectomy in recent studies. However, no prospective studies comparing laparoendoscopic single-site (LESS) and vNOTES hysterectomy have been performed. This study aims to evaluate postoperative pain and safety of vNOTES hysterectomy compared to LESS hysterectomy for benign uterine disease.

Methods: This study is a prospective, investigator-initiated randomized controlled pilot trial. A total of 26 patients were randomized and allocated to vNOTES group $(n=13)$ and LESS group $(n=13)$. The primary outcome was postoperative abdominal and vaginal pain evaluated $24 \mathrm{~h}$ after surgery. Secondary outcomes included the number of additional
\end{abstract}

S. J. Park · H. S. Kim $(\bowtie)$

Department of Obstetrics and Gynecology, Seoul

National University College of Medicine, 101

Daehak-Ro Jongno-Gu, 110-744, Seoul 03080,

Republic of Korea

e-mail: bboddi0311@gmail.com

G. W. Yim $(\bowtie)$

Department of Obstetrics and Gynecology,

Dongguk University College of Medicine, 27

Dongguk-ro, Ilsandong-gu, Goyang, Gyeonggi

10326, Republic of Korea

e-mail: gawonyim@gmail.com analgesics administered and the maintenance rate of patient-controlled analgesia (PCA).

Results: No differences were shown in baseline characteristics between the two groups. Operation time was longer in the LESS group (median, 55 vs. $75 \mathrm{~min} ; P=0.027$ ), and there were no differences in estimated blood loss, postoperative hemoglobin level, surgical indications, and hospitalization days. Postoperative abdominal pain intensity did not differ between the two groups, while the vNOTES group showed higher postoperative vaginal pain than the LESS group at 16 and $24 \mathrm{~h}$ after surgery (median, 3 vs. 1 and 2 vs. $0, P=0.007$ and $P=0.010$, at 16 and $24 \mathrm{~h}$ respectively). No differences were shown in the number of additional analgesics and PCA use between the two groups.

Conclusions: vNOTES hysterectomy can be safely performed for benign uterine disease requiring hysterectomy. However, vNOTES hysterectomy might be associated with higher postoperative vaginal pain intensity compared to LESS hysterectomy.

Trial Registration: CRIS identifier KCT0004605.

Keywords: Hysterectomy; Natural orifice transluminal endoscopic surgery; Postoperative pain; Laparoscopy 


\section{Key Summary Points}

Why carry out this study?

Vaginal natural orifice transluminal endoscopic surgery (vNOTES) approach for benign uterine disease is feasible and safe according to previous studies, but the postoperative pain reduction effect of vNOTES is controversial.

This study aims to evaluate whether vNOTES could reduce postoperative pain compared to laparoendoscopic single-site (LESS) surgery on women requiring hysterectomy for benign disease.

\section{What was learned from the study?}

Postoperative abdominal pain did not differ between the two groups, but vaginal pain was higher in the vNOTES group compared to the LESS group.

vNOTES may not reduce postoperative pain, but further study is needed because the patient-controlled analgesia usage may have masked the postoperative pain difference.

\section{INTRODUCTION}

Vaginal natural orifice transluminal endoscopic surgery (vNOTES) approach is familiar to gynecologic surgeons proficient in vaginal surgeries. Several study groups demonstrated the safety of vNOTES hysterectomy compared to conventional laparoscopic hysterectomy [1-5]. Furthermore, the possibility of vNOTES having less postoperative pain than laparoscopic surgery has also been suggested [2-7]. However, previous studies have mainly compared multiport total laparoscopic hysterectomy to vNOTES, which limits the exact comparison because of the difference in the location and number of trocar entry sites [1, 2, 4-7]. There has been only a single retrospective study that compared single-port laparoscopic-assisted vaginal hysterectomy with vNOTES, which showed no advantage in pain or surgical outcomes except shorter operative time and hospital stay in the vNOTES group [3].

A recent systematic review and meta-analysis that compared vNOTES hysterectomy to laparoendoscopic single-site (LESS) approach for benign indications yielded outcomes of one randomized controlled trial and five retrospective cohort trials [8]. Although vNOTES hysterectomy showed significantly shorter operation time and decrease in estimated blood loss, there were no differences in operative complications and postoperative pain scores. Likewise, clinical outcomes including the pain profiles of vNOTES hysterectomy compared to LESS surgery vary among studies and are largely unknown, which warrants a prospective clinical trial.

As minimally invasive surgical methods, LESS and vNOTES hysterectomy are assumed to be both cosmetically advantageous in terms of appearance because there is no scar. However, postoperative pain and safety evaluations have not been prospectively evaluated. We hypothesized that vNOTES hysterectomy may reduce postoperative pain compared to LESS hysterectomy. Therefore, we designed a randomized controlled pilot study to evaluate postoperative pain and safety after LESS and vNOTES hysterectomy for benign uterine disease.

\section{METHODS}

\section{Study Design}

This study was a prospective, investigator-initiated randomized controlled pilot trial to evaluate the postoperative pain difference and clinical outcomes in vNOTES and LESS hysterectomy. Seoul National University Institutional Review Board approved the study (H1909-137-1066), which was registered on the Clinical Research Information Service (CRIS) registry before the first patient enrollment (CRIS identifier KCT0004605). This study was performed in accordance with the Helsinki 
Declaration of 1964 and its later amendments, and all participants signed the informed consent form.

\section{Sample Size}

This study was designed as a pilot study because the postoperative pain score data in vNOTES and LESS hysterectomy have not been investigated in previous studies, and for this reason, 12 participants were assigned in each group [9]. Considering the dropout rate of $10 \%$, we planned to enroll a total of 26 patients.

\section{Participants}

Women who required hysterectomy because of benign uterine disease or abnormal uterine bleeding were screened for this study. The inclusion criteria were as follows: women of age 19 years or more and under the age of 80 years; suitable for endoscopic surgery evaluated by the American Society of Anesthesiologists Physical Status (ASA) classification 1 or 2; signed to the informed consent form. The exclusion criteria were as follows: age of less than 19 years or over the age of 80 years; pregnant or breastfeeding; no history of sexual intercourse; suspected of pelvic malignancy; women who have received three or more cesarean sections; suspected of posterior cul de sac obliteration by gynecological examination.

\section{Randomization}

A reproducible randomization table was generated using a web-based program and managed by a third-party gynecologist unrelated to the study. Participants who meet the eligibility criteria were sequentially assigned to the experimental and control group at a ratio of $1: 1$ immediately before surgery. The randomization results were disclosed to both the investigator and the study subjects because of the nature of this study. The patients allocated to the experimental group underwent vNOTES hysterectomy (vNOTES group), while the patients allocated to the control group underwent LESS hysterectomy (LESS group).

\section{Procedures}

All surgeries were performed by an expert gynecologist (HSK) according to a consistent standard operating procedure. For vNOTES patients, hysterectomy was performed according to the procedure reported previously [10]. First, the vaginal incision was made around the cervix. Then, the vesicouterine and the rectouterine pouch were developed, and bilateral uterosacral ligaments were incised. After insertion of One Port Plus ${ }^{\circledR}$ (MEDIFINE Corporation Co., Ltd, Gyeonggi-do, Korea) through the vagina, carbon dioxide $\left(\mathrm{CO}_{2}\right)$ gas was infused with a pressure of $12 \mathrm{mmHg}$. A $10-\mathrm{mm}$ rigid $0^{\circ}$ laparoscope was used (Karl Storz, Tuttlingen, Germany), and both parametrium and uterine arteries were coagulated and cut with the energy device. After the hysterectomy was completed, vaginal cuff closure was done extracorporeally with continuous locking suture with $1-0$ vicryl.

Patients allocated to the control group underwent LESS hysterectomy. A single umbilical incision of $2 \mathrm{~cm}$ was made, and the surgeon used the open technique to approach the peritoneal cavity. One Port Plus was inserted to the umbilical incision, and a $5-\mathrm{mm}$ rigid $30^{\circ}$ laparoscope (Karl Storz, Tuttlingen, Germany) was used. Hysterectomy was performed, and the specimen was withdrawn through the vagina. After that, vaginal cuff closure was done intracorporeally with barbed suture.

For all participants, salpingectomy was done after preoperative counseling, and concomitant oophorectomy was performed in menopausal women. Anesthesiologists applied patient-controlled analgesia (PCA) after surgery upon patient consent. PCA was composed of $1000 \mu \mathrm{g}$ of fentanyl without basal flow. Bolus amount was $10 \mu \mathrm{g}$ with lockout time of $6 \mathrm{~min}$. Postoperative pain was evaluated on the basis of the written nursing protocol developed for this trial. The nursing staff assessed the patients' abdominal and vaginal pain intensity immediately after arriving at the ward and every $8 \mathrm{~h}$ until discharge using the numeric rating scale (NRS). Abdominal pain refers to pain on the abdomen and pelvis, and vaginal pain refers to pain in the perineal area and both sites were 
assessed separately according to the patients' pain complaints. Patients were asked to rate their pain intensity on a scale from 0 to 10 , where 0 represents no pain at all and 10 being the worst pain possible [11]. If the pain score was 4 or more at any site by NRS [12], $50 \mathrm{mg}$ of tramadol was immediately administered by an intravenous route. When the pain did not improve upon reevaluation after $30 \mathrm{~min}$, an additional nonsteroidal anti-inflammatory drug (dexketoprofen $50 \mathrm{mg}$ ) was administered intravenously. In patients complaining of side effects (dizziness, nausea) due to PCA infusion, its use was discontinued upon patient request. Pain evaluation and management were performed in the same manner regardless of PCA use. Nonsteroidal anti-inflammatory drug (zaltoprofen $80 \mathrm{mg}$ ) was given orally three times daily from $24 \mathrm{~h}$ after surgery and for 7 days thereafter.

\section{Outcome Measures}

The primary outcome was postoperative abdominal and vaginal pain at $24 \mathrm{~h}$ after surgery by NRS score. The secondary outcome regarding the postoperative pain included the NRS pain score recorded every $8 \mathrm{~h}$ up to $48 \mathrm{~h}$ post-surgery, the frequency of additional analgesics administered, and maintenance rate of PCA at the time of the postoperative pain evaluation. Also, we measured the estimated blood loss, transfusion, postoperative hemoglobin level at $24 \mathrm{~h}$ after surgery, days of hospitalization, postoperative complications, and the rate of reoperation within 6 weeks.

\section{Statistical Analysis}

We analyzed categorical variables between two groups using the chi-square test. Non-parametric variables of two groups were analyzed using the Mann-Whitney $U$ test. All statistical analysis was regarded as significant in cases of $P<0.05$, and SPSS software version 23.0 (SPSS INC., Chicago, IL, USA) was used for analysis.

\section{RESULTS}

\section{Participants}

A total of 29 patients were assessed for eligibility from February 2020 to September 2020. Finally, 26 patients were randomized into the control and experimental groups, and all 26 patients were included for analysis in the per-protocol population (Fig. 1). There were no differences in age, ASA score, indications for surgery, parity, and the number of previous cesarean sections; however, body mass index (BMI) showed a difference between the two groups (median, 23.8 vs. $21.8 ; P=0.044$; Table 1 ).

\section{Operative Outcomes}

In terms of operative outcomes, the vNOTES group showed a shorter operation time than the LESS group (median, 55 vs. $75 \mathrm{~min} ; P=0.027$ ). Otherwise, there were no differences in the estimated blood loss, transfusion rate, preoperative and postoperative hemoglobin level, operation type, final pathologic diagnosis, and postoperative complications. Also, we documented the cases of vaginal orifice laceration that required simple suturing during surgery, which showed no difference between the two groups. Furthermore, the number of additional analgesics administered after surgery showed no differences. All patients allocated to each group completed the planned surgical procedure (Table 2).

\section{Postoperative Pain}

Table 3 and Fig. 2 show postoperative pain outcomes. In terms of abdominal pain, there were no differences in NRS scores among the two groups. However, postoperative vaginal pain score was higher in vNOTES group at 16 and $24 \mathrm{~h}$ after surgery (median, range; $3,0-5$ vs. $1,0-4$ and $2,0-3$ vs. $0,0-2, P=0.007$ and $P=0.010$, at 16 and $24 \mathrm{~h}$ respectively). We performed subgroup analysis for patients who did not undergo additional vaginal suturing for laceration, and as a result, vaginal postoperative pain score was also higher in the vNOTES group 


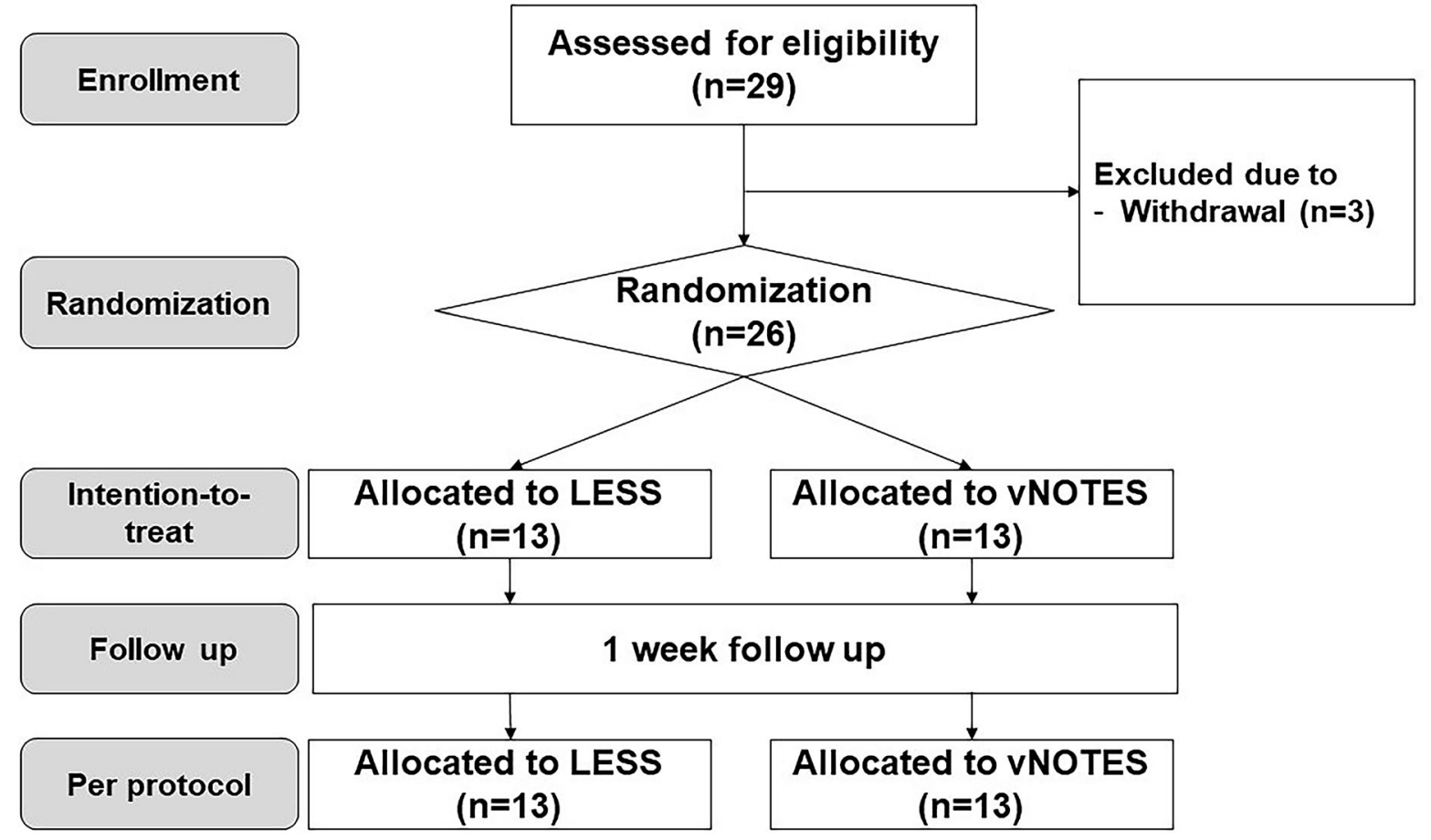

Fig. 1 Flowchart of the study

at 16 and $24 \mathrm{~h}$ after surgery (median, range; 3 , $0-5$ vs. $0,0-1$ and $0,1-3$ vs. $0,0-1, P=0.008$ and $P=0.013$, at 16 and $24 \mathrm{~h}$ respectively). There was no difference in the proportion of patients with PCA maintenance at each evaluation point.

\section{DISCUSSION}

This randomized pilot study evaluated postoperative pain and surgical outcomes of vNOTES and LESS hysterectomy. vNOTES hysterectomy had shorter operation time compared to LESS hysterectomy. However, LESS hysterectomy showed higher postoperative vaginal pain score at 16 and $24 \mathrm{~h}$ after surgery. To the best of our knowledge, this is the first prospective randomized controlled pilot study comparing LESS and vNOTES hysterectomy. Regarding vNOTES hysterectomy, the HALON trial by Baekelandt and colleagues is the only prospective randomized trial comparing total laparoscopic and vNOTES hysterectomy [4]. There is only a single retrospective study that compared single-port laparoscopic-assisted vaginal and vNOTES hysterectomy [3], and other studies have reported retrospective comparison data of multiport laparoscopic and vNOTES hysterectomy $[1,2,5]$.

The major findings of this study are as follows. First, the operation time was found to be shorter in the vNOTES group in this trial. Previous reports have shown consistent outcomes of shorter operation time in vNOTES hysterectomy than laparoscopic hysterectomy [3-7]. Shorter operation time in vNOTES might be associated with the time reduced by omitting open and closure procedures of the umbilicus. Also, compared to the umbilical incision with the approximate size of about $2 \mathrm{~cm}$, the vNOTES approach may allow more expansive working space, resulting in less collision of the laparoscopic instruments which may contribute to shortened operation time.

Second, there were no differences in postoperative abdominal pain up to $48 \mathrm{~h}$ after surgery. This result is different from that of the HALON trial, which showed consistent and significantly higher postoperative pain in the 
Table 1 Demographic and clinical characteristics of participants

\begin{tabular}{|c|c|c|c|}
\hline Characteristics & vNOTES $(n=13)$ & LESS $(n=13)$ & $P$ value \\
\hline Age, median (range) & $54(35-77)$ & $48(34-63)$ & 0.081 \\
\hline BMI, median (range) & $23.8(21.32-27.6)$ & $21.8(20.2-25.9)$ & 0.044 \\
\hline \multicolumn{4}{|l|}{ ASA score, $n(\%)$} \\
\hline 1 & $1(7.7 \%)$ & $2(15.4 \%)$ & \multirow[t]{3}{*}{0.513} \\
\hline 2 & $11(84.6 \%)$ & $11(84.6 \%)$ & \\
\hline 3 & $1(7.7 \%)$ & 0 & \\
\hline \multicolumn{4}{|c|}{ Indication for surgery, $n$ (\%) } \\
\hline Myoma & $7(53.8 \%)$ & $8(61.5 \%)$ & \multirow[t]{6}{*}{0.369} \\
\hline CIN3 & $3(23.1 \%)$ & $1(7.7 \%)$ & \\
\hline Adenomyosis & $2(15.4 \%)$ & 0 & \\
\hline Endometrial hyperplasia & $1(7.7 \%)$ & $2(15.4 \%)$ & \\
\hline AIS & 0 & $1(7.7 \%)$ & \\
\hline AUB & 0 & $1(7.7 \%)$ & \\
\hline \multicolumn{4}{|l|}{ Parity, $n(\%)$} \\
\hline 0 & $1(7.7 \%)$ & $4(30.8 \%)$ & \multirow[t]{4}{*}{0.370} \\
\hline 1 & $3(23.1 \%)$ & $4(30.8 \%)$ & \\
\hline 2 & $6(46.2 \%)$ & $3(23.1 \%)$ & \\
\hline 3 or more & $3(23.1 \%)$ & $2(15.4 \%)$ & \\
\hline \multicolumn{4}{|c|}{ Previous cesarean section history, $n$ (\%) } \\
\hline 0 & $9(69.2 \%)$ & $10(76.9 \%)$ & \multirow[t]{4}{*}{0.824} \\
\hline 1 & $2(15.4 \%)$ & $2(15.4 \%)$ & \\
\hline 2 & $2(15.4 \%)$ & $1(7.7 \%)$ & \\
\hline 3 or more & 0 & 0 & \\
\hline
\end{tabular}

vNOTES vaginal natural orifice transluminal endoscopic surgery, LESS laparoendoscopic single-site, BMI body mass index, $A S A$ American Society of Anesthesiologists physical status classification system, CIN cervical intraepithelial neoplasia, AIS adenocarcinoma in situ, $A U B$ abnormal uterine bleeding

total laparoscopic hysterectomy group from immediately after surgery to the 7 th postoperative day [4]. This difference can be explained by the general use of PCA in our study participants which may have hindered the difference in postoperative abdominal pain intensity between the two surgical methods. PCA was not used in the HALON trial [4].
Third, postoperative vaginal pain was higher in the vNOTES group than in the LESS group. This is the first study to analyze vaginal pain separately from abdominal pain. Contrary to the findings of this study, several studies have reported significantly lower postoperative pain in the vNOTES group than in the laparoscopy group $[2,4,8,13]$. On the other hand, previous 
Table 2 Surgical outcomes

\begin{tabular}{|c|c|c|c|}
\hline Outcome variables & vNOTES $(n=13)$ & LESS $(n=13)$ & $P$ value \\
\hline Operation time, mins (range) & $55(25-105)$ & $75(50-110)$ & 0.027 \\
\hline Estimated blood loss, $\mathrm{mL}$ (range) & $100(0-650)$ & $100(0-300)$ & 0.687 \\
\hline Transfusion, $n$ (range) & $0(0-3)$ & $0(0-0)$ & 0.511 \\
\hline Preoperative hemoglobin, g/dL (range) & $13.1(9-14.4)$ & $12.3(11.114 .1)$ & 0.448 \\
\hline Postoperative hemoglobin, g/dL (range) & $11.6(7.8-13.8)$ & $10.8(8.6-14.0)$ & 0.920 \\
\hline Hospitalization, days (range) & $4(4-4)$ & $4(4-5)$ & 0.762 \\
\hline \multicolumn{4}{|l|}{ Operation type, $n(\%)$} \\
\hline Hysterectomy & $1(7.7 \%)$ & $2(15.4 \%)$ & 0.721 \\
\hline Hysterectomy + unilateral salpingo-oophorectomy & $1(7.7 \%)$ & 0 & \\
\hline Hysterectomy + bilateral salpingo-oophorectomy & $6(46.2 \%)$ & $6(46.2 \%)$ & \\
\hline Hysterectomy + bilateral salpingectomy & $5(38.5 \%)$ & $5(38.5 \%)$ & \\
\hline \multicolumn{4}{|l|}{ Diagnosis, $n(\%)$} \\
\hline Myoma & $7(53.8 \%)$ & $7(53.8 \%)$ & 0.544 \\
\hline CIN3 & $3(23.1 \%)$ & $1(7.7 \%)$ & \\
\hline Endometrial hyperplasia & $1(7.7 \%)$ & $1(7.7 \%)$ & \\
\hline Adenomyosis & $1(7.7 \%)$ & $1(7.7 \%)$ & \\
\hline AIS & 0 & $1(7.7 \%)$ & \\
\hline Endometrial cancer & 0 & $2(15.4 \%)$ & \\
\hline Cervical cancer & $1(7.7 \%)$ & 0 & \\
\hline Size of the uterus, $\mathrm{cm}$, (range) & $9.5(5.1-17)$ & $9.5(7-15)$ & 0.7 \\
\hline Weight of the uterus, g, (range) & $238(40.8-940)$ & $196(93-346)$ & 0.98 \\
\hline Postoperative complication, $n(\%)$ & & & 0.593 \\
\hline Bleeding & $1(7.7 \%)$ & $1(7.7 \%)$ & \\
\hline Surgical site infection & $1(7.7 \%)$ & 0 & \\
\hline Vaginal laceration or suture, $n(\%)$ & $4(30.8 \%)$ & $3(23.1 \%)$ & 0.658 \\
\hline Number of additional analgesics, median (range) & $1(0-3)$ & $1(0-3)$ & 0.647 \\
\hline
\end{tabular}

vNOTES vaginal natural orifice transluminal endoscopic surgery, LESS laparo-endoscopic single-site, CIN cervical intraepithelial neoplasia, $A I S$ adenocarcinoma in situ 


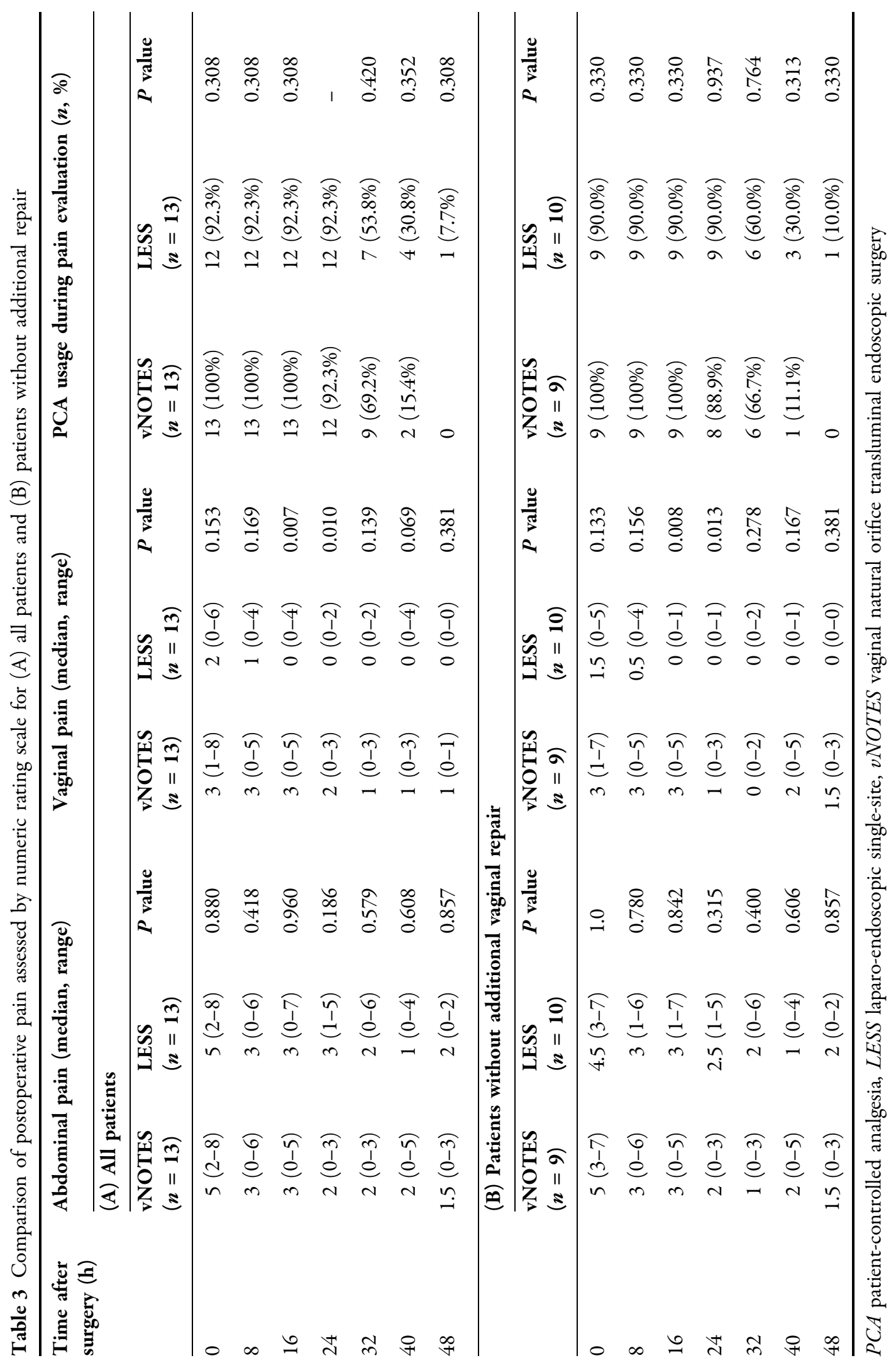


(i)

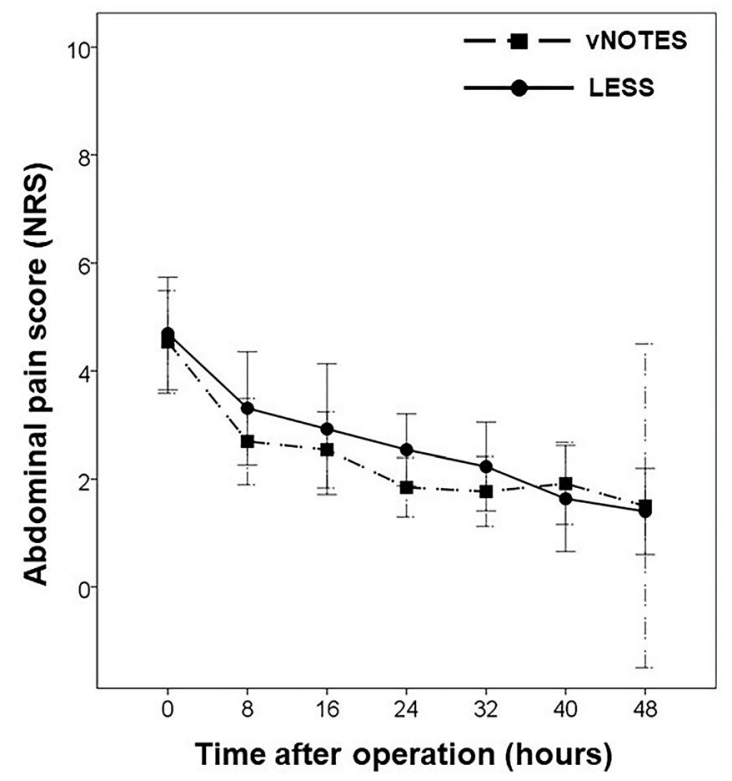

(ii)

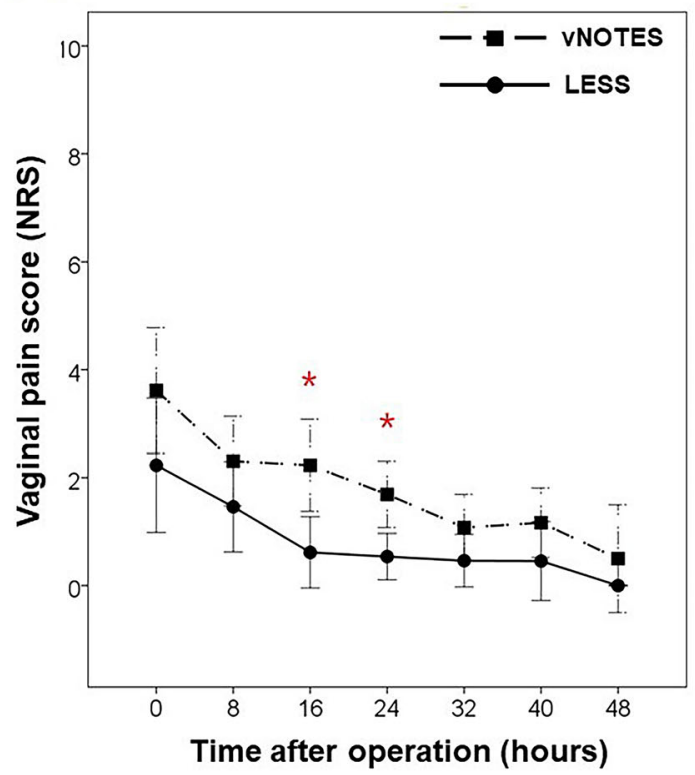

Fig. 2 Postoperative (i) abdominal pain and (ii) vaginal pain by numeric rating scale up to $48 \mathrm{~h}$ after the operation

studies that evaluated postoperative pain between laparoscopic and vaginal hysterectomy have reported higher postoperative pain in vaginal hysterectomy $[12,14]$. Although vaginal hysterectomy had been generally considered the most minimally invasive surgery and with the least postoperative pain, these studies showed higher postoperative pain after vaginal hysterectomy. It is hypothesized that the usage of vessel sealing devices in vNOTES rather than suture and ligation as in vaginal hysterectomy might have reduced postoperative pain compared to laparoscopic hysterectomy $[15,16]$. In our surgical procedure during vNOTES, we applied additional suturing between the uterine artery and vaginal wall for bleeding control and vaginal cuff closure. Nevertheless, pain scores at $16 \mathrm{~h}$ and $24 \mathrm{~h}$ after surgery were less than 4 , not requiring additional analgesics. The result of this study suggests that both LESS and vNOTES operations are clinically tolerable in terms of postoperative pain.

A limitation of our study is the relatively small sample size due to the nature of the pilot study intended to estimate the postoperative pain score for further clinical trial. Also, the usage of PCA might have hindered the ability to show a difference in the postoperative pain score. At our institution, we give patients the option of PCA routinely before surgery and most patients choose to be under postoperative PCA, as shown in this trial. Nonetheless, considering the possibility that the use of PCA interfered with the evaluation of postoperative pain, future studies regarding postoperative pain in vNOTES may consider eliminating the PCA effect in the study protocol. Finally, a relatively high rate of additional suturing for vaginal laceration might have affected the primary outcome measures, despite no differences shown in the subgroup analysis.

\section{CONCLUSIONS}

vNOTES can be safely performed for benign uterine disease requiring hysterectomy. Compared to LESS approach, vNOTES hysterectomy might be associated with higher postoperative vaginal pain which requires further investigation. 


\section{ACKNOWLEDGEMENTS}

We thank the participants of the study. We also deeply appreciate MEDIFINE Corporation Co., Ltd (Gyeonggi-do, Republic of Korea) for its collaborative work. However, it had no role in study design, data collection and analysis, decision to publish, Rapid Service Fee, or preparation of the manuscript.

Funding. This study was supported by the grant from Seoul National University Hospital (No. 0620194870). The Rapid Service Fee was funded by the authors.

Authorship. All named authors meet the International Committee of Medical Journal Editors (ICMJE) criteria for authorship for this article, take responsibility for the integrity of the work as a whole, and have given their approval for this version to be published.

Authors' Contributions. Soo Jin Park: conceptualization, methodology, formal analysis and investigation, writing original draft preparation, and resources. Hee Seung Kim: conceptualization, methodology, formal analysis and investigation, funding acquisition, resources, and supervision. Ga Won Yim: conceptualization, methodology, writing original draft preparation, resources, and supervision.

Disclosures. Dr. Soo Jin Park, Hee Seung Kim and Ga Won Yim have no conflicts of interest or financial ties to disclose.

Compliance with Ethics Guidelines. This study was approved from Seoul National University Institutional Review Board (H-1909137-1066), and performed in accordance with the Helsinki Declaration of 1964, and its later amendments. All subjects signed informed consent form and their identifying information will not be included in the manuscript.

Data Availability. The datasets generated during and/or analyzed during the current study are available from the corresponding author on reasonable request.
Open Access. This article is licensed under a Creative Commons Attribution-NonCommercial 4.0 International License, which permits any non-commercial use, sharing, adaptation, distribution and reproduction in any medium or format, as long as you give appropriate credit to the original author(s) and the source, provide a link to the Creative Commons licence, and indicate if changes were made. The images or other third party material in this article are included in the article's Creative Commons licence, unless indicated otherwise in a credit line to the material. If material is not included in the article's Creative Commons licence and your intended use is not permitted by statutory regulation or exceeds the permitted use, you will need to obtain permission directly from the copyright holder. To view a copy of this licence, visit http://creativecommons.org/licenses/by$\mathrm{nc} / 4.0 /$.

\section{REFERENCES}

1. Kim $\mathrm{SH}$, Jin $\mathrm{CH}$, Hwang IT, et al. Postoperative outcomes of natural orifice transluminal endoscopic surgery-assisted vaginal hysterectomy and conventional laparoscopic-assisted vaginal hysterectomy: a comparative study. Obstet Gynecol Sci. 2018;61(2):261.

2. Yang C-Y, Shen T-C, Lin C-L, Chang Y-Y, Huang C-C, Lin W-C. Surgical outcomes of hysterectomy by transvaginal natural orifice transluminal endoscopic surgery (vNOTES) compared with laparoscopic total hysterectomy (LTH) in women with non-prolapsed and benign uterine diseases. Taiwan J Obstet Gynecol. 2020;59(4):565-9.

3. Yang YS, Kim SY, Hur MH, Oh KY. Natural orifice transluminal endoscopic surgery-assisted versus single-port laparoscopic-assisted vaginal hysterectomy: a case-matched study. J Minim Invasive Gynecol. 2014;21(4):624-31.

4. Baekelandt J, De Mulder P, Le Roy I, et al. Hysterectomy by transvaginal natural orifice transluminal endoscopic surgery versus laparoscopy as a day-care procedure: a randomised controlled trial. BJOG. 2019;126(1):105-13.

5. Wang C-J, Huang H-Y, Huang C-Y, Su H. Hysterectomy via transvaginal natural orifice transluminal endoscopic surgery for nonprolapsed uteri. Surg Endosc. 2015;29(1):100-7. 
6. Kaya C, Alay I, Cengiz H, Yıldız GO, Baghaki HS, Yasar L. Comparison of hysterectomy cases performed via conventional laparoscopy or vaginally assisted natural orifice transluminal endoscopic surgery: a paired sample cross-sectional study. J Obstet Gynaecol. 2021;41(3):434-8.

7. Kaya C, Yıldız Ş, Alay İ, Aslan Ö, Aydıner İE, Yaşar L. The comparison of surgical outcomes following laparoscopic hysterectomy and vNOTES hysterectomy in obese patients. J Ivest Surg. 2021:1-6. https://doi.org/10.1080/08941939.2021.1927262.

8. Housmans S, Noori N, Kapurubandara S, et al. Systematic review and meta-analysis on hysterectomy by vaginal natural orifice transluminal endoscopic surgery (vNOTES) compared to laparoscopic hysterectomy for benign indications. J Clin Med. 2020;9(12):3959.

9. Julious SA. Sample size of 12 per group rule of thumb for a pilot study. Pharm Stat. 2005;4(4): 287-91.

10. Oh SH, Park SJ, Lee EJ, Yim GW, Kim HS. Pelvic lymphadenectomy by vaginal natural orifice transluminal endoscopic surgery (vNOTES) for earlystage endometrial cancer. Gynecol Oncol. 2019;153(1):211-2.

11. Gerbershagen HJ, Rothaug J, Kalkman C, Meissner W. Determination of moderate-to-severe postoperative pain on the numeric rating scale: a cut-off point analysis applying four different methods. $\mathrm{Br} \mathrm{J}$ Anaesth. 2011;107(4):619-26.

12. Ghezzi F, Uccella S, Cromi A, et al. Postoperative pain after laparoscopic and vaginal hysterectomy for benign gynecologic disease: a randomized trial. Am J Obstet Gynecol. 2010;203(2):118.

13. Yang E, Nie D, Li Z. Comparison of major clinical outcomes between transvaginal notes and traditional laparoscopic surgery: a systematic review and meta-analysis. J Surg Res. 2019;244:278-90.

14. Pokkinen SM, Kalliomäki M-L, Yli-Hankala A, Nieminen K. Less postoperative pain after laparoscopic hysterectomy than after vaginal hysterectomy. Arch Gynecol Obstet. 2015;292(1):149-54.

15. Silva-Filho AL, Rodrigues AM, de Castro Monteiro $\mathrm{MV}$, et al. Randomized study of bipolar vessel sealing system versus conventional suture ligature for vaginal hysterectomy. Eur J Obstet Gynecol Reprod Biol. 2009;146(2):200-3.

16. Hefni M, Bhaumik J, El-Toukhy T, et al. Safety and efficacy of using the LigaSure vessel sealing system for securing the pedicles in vaginal hysterectomy: randomised controlled trial. BJOG. 2005;112(3): 329-33. 